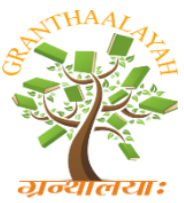

INTERNATIONAL JOURNAL OF RESEARCH GRANTHAALAYAH A knowledge Repository

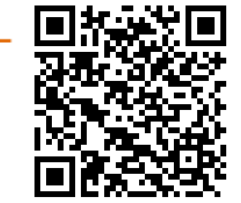

Science

\title{
CURRENT VOLTAGE CHARACTERISTIC OF PLANAR LANGMUIR PROBE IN IONOSPHERIC MAXWELLIAN PLASMA
}

\author{
Shankar Bhattarai ${ }^{* 1}$, Lekha Nath Mishra ${ }^{2}$ \\ ${ }^{1}$ MSc Physics, Department of Physics, Patan Multiple Campus, Tribhuvan University, Lalitpur, \\ Nepal \\ ${ }^{2}$ Assoc. Prof., Department of Physics, Patan Multiple Campus, Tribhuvan University, Lalitpur, \\ Nepal
}

DOI: https://doi.org/10.29121/granthaalayah.v5.i4.2017.1815

\begin{abstract}
Frequently used geometries of Langmuir probes are planar, spherical, and cylindrical shapes. The geometry is chosen depending on the purpose of the measurements and the platform configuration. Planar Langmuir Probes have been installed on satellites and sounding rockets to observe the general characteristics of thermal plasma in the ionosphere for more than five decades. Because of its simplicity and convenience, the Langmuir probe is one of the most frequently installed scientific instruments on spacecraft. The Planar Langmuir Probe is the key plasma diagnostic used by scientists interested in plasma characterization to measure the internal parameters of the bulk of the plasma. This research explores the theoretical study of Planar Langmuir Probe I-V Characteristics. The relationship between first derivative of current verses applied probe voltage is also computed. With the help of the (volt-ampere curves) of Planar Langmuir probes, the different parameters of plasma can be determined such as plasma potential, floating potential, probe currents in different probe voltage and so on. Planar Langmuir probe geometry is easy to construct and equally suitable for plasma characterization.
\end{abstract}

Keywords: Planar Probe; Plasma Parameters; I-V Characteristic; Maxwellian Plasma.

Cite This Article: Shankar Bhattarai, and Lekha Nath Mishra. (2017). "CURRENT VOLTAGE CHARACTERISTIC OF PLANAR LANGMUIR PROBE IN IONOSPHERIC MAXWELLIAN PLASMA." International Journal of Research - Granthaalayah, 5(4), 228-237. https://doi.org/10.29121/granthaalayah.v5.i4.2017.1815.

\section{Introduction}

The electromagnetic force is generally observed to create structure: e.g., stable atoms and molecules, crystalline solids. In fact, the most widely studied consequences of the electromagnetic force form the subject matter of Chemistry and Solid-State Physics, which are both disciplines developed to understand essentially static structures. Structured systems have 
binding energies larger than the ambient thermal energy. Placed in a sufficiently hot environment, they decompose: e.g., crystals melt, molecules disassociate. At temperatures near or exceeding atomic ionization energies, atoms similarly decompose into negatively charged electrons and positively charged ions. These charged particles are by no means free: in fact, they are strongly affected by each other's electromagnetic fields. Nevertheless, because the charges are no longer bound, their assemblage becomes capable of collective motions of great vigor and complexity. Such an assemblage is termed a plasma.

The Czech medical scientist, Johannes Evangelista Purkinje introduced use of the Greek word plasma (meaning 'formed or molded') in the mid-19 ${ }^{\text {th }}$ century, to denote the clear fluid which remains after removal of all the corpuscular material in blood. The Nobel Prize winning American chemist Irving Langmuir first used this term to describe an ionized gas in 1927 [1]. The term plasma means something molded or fabricated and comes from Greek. As the temperature of the material is raised, its state changes from solid to liquid and then liquid to gas. If the temperature is increased further-more, the molecule will dissociate into atoms and ultimately some of their electrons become so energetic that the atom ionizes. At these high temperatures, the gas becomes a mixture of oppositely charged particles and neutral atoms. Thus, a gas which contains large number of ionized particles and neutral atoms which show quasineutrality and collective behavior are called plasma. In short, "Plasma is a quasi-neutral gas of charged and neutral particles which exhibits collective behavior".

Electric probes, such as the Langmuir probe, are the most widely used plasma diagnostic tools [2]. Other methods include passive remote sensing methods, which detects radiation or particles emitted spontaneously by the plasma, such as studying plasma phenomena related to the sun active non-contact methods, where radiations or particles produced by external sources, such as the study of the absorption and scattering of electromagnetic radiation by the plasma [3]. Langmuir probing determines the properties of a plasma with excellent spatial resolution, enabling the reconstruction of the crossing point of the particle [4]. Additionally, this method successfully describes the plasma's parameters in a variety of situations, such as low-pressure plasmas for material processing, edge plasmas in fusion devices, and ionospheric plasma and it aids the design of ion sources and new plasma chambers.

The simplest application of a Langmuir Probe involves inserting the probe into the plasma region of interest and applying a voltage $V$ with respect to the metallic chamber that contains the plasma. Several configurations are commonly used, including single, double, and triple probes [5]. These variations allow for simplifications in the control system or in the analysis of measurements. The bias voltage is typically swept at a specified frequency over a range that is comparable to the electron temperature to determine the current-voltage (I-V) characteristic for the local plasma. The plasma parameters are extracted during a post-processing step, where software can be used to fit the I-V characteristics.

The Langmuir probe technique involves applying a voltage to a metallic conductor immersed in plasma and observing the collected current. The observed total current, $I$, is a summation of various currents such as electron and ion thermal currents, photoelectron current, secondary electron currents, etc [6]. The benefits of the simplicity of this technique, however, are offset by the complexity of the theory required to analyze the obtained current vs. potential, or $I$ - $V$, curves. 
There is no general theory of Langmuir probes which is applicable to all measurement conditions, because it depends on the probe size and geometry, plasma density and temperature, platform velocity, and other factors. The actual design of the probe is usually determined by considering the relationship between the probe dimensions and the Debye length of the plasma. In general, two approximations are used to express the current on the probe in the plasma: 1) orbital motion limited (OML) and 2) sheath area limited (SAL). OML theory can be adopted when the probe radius is smaller than the thickness of the sheath surrounding the probe, while it must be equal to or larger than the sheath thickness in the case of SAL theory [7].

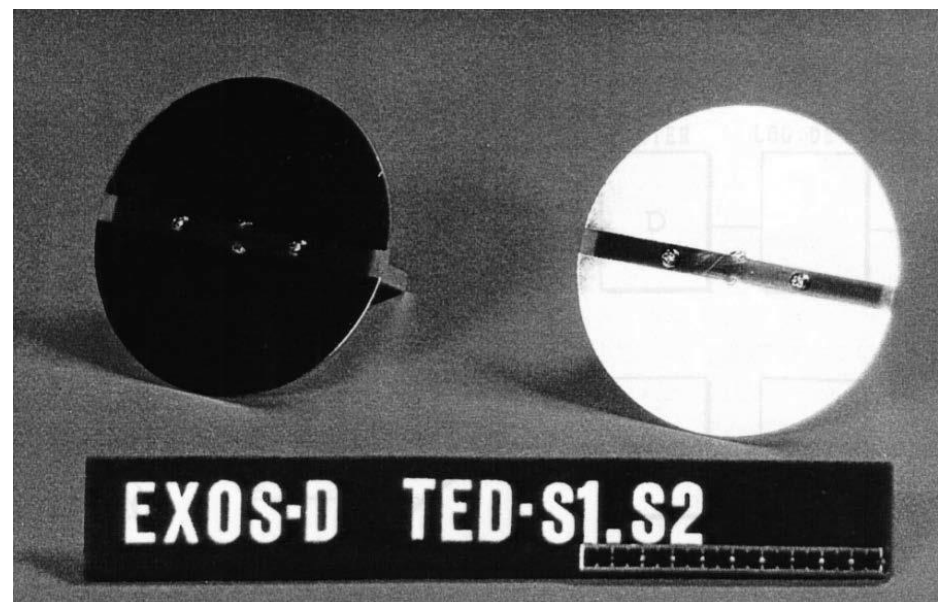

Figure1: Planar probe installed on Japanese Akebono satellite.

Langmuir probes may have different electrode shapes, such as cylindrical, spherical and planar. Planar Langmuir probes are not that common. The main disadvantage of them is that it is not easy to neglect boundary effects, in order to model them as an infinite planar conductor. This could be done by making the probe large enough, compared to the Debye length. Nevertheless, this approach is not always acceptable in laboratory and industrial plasmas, as it would most likely cause an equally large perturbation in the plasma that is to be diagnosed, which is an undesirable effect. However, there are situations where this geometry may come in handy, e. $g$. probes designed to be mounted on diverter plates in fusion devices, where the probe has to overcome huge heat fluxes. Anyway, fusion devices are not the only place where planar probes are used [8].

\section{Current-Voltage Characteristics of Langmuir Probe}

The exact shape of the current to voltage $(I-V)$ characteristic curve of a Langmuir probe, depends on the properties of the plasma where it is being used, as well as on the geometry and other attributes of the probe itself. Nevertheless, the $I-V$ characteristic curve of any Langmuir probe can be qualitatively described as the one that appears in Fig. below. The diagnosis of a plasma is based on the comparison of the current, in one zone or point of the $I-V$ characteristic curve, with the prediction of a particular theoretical model. In this way, the current and biasing voltage of the probe are correlated to properties of the plasma, as the electron density or temperature [9]. 


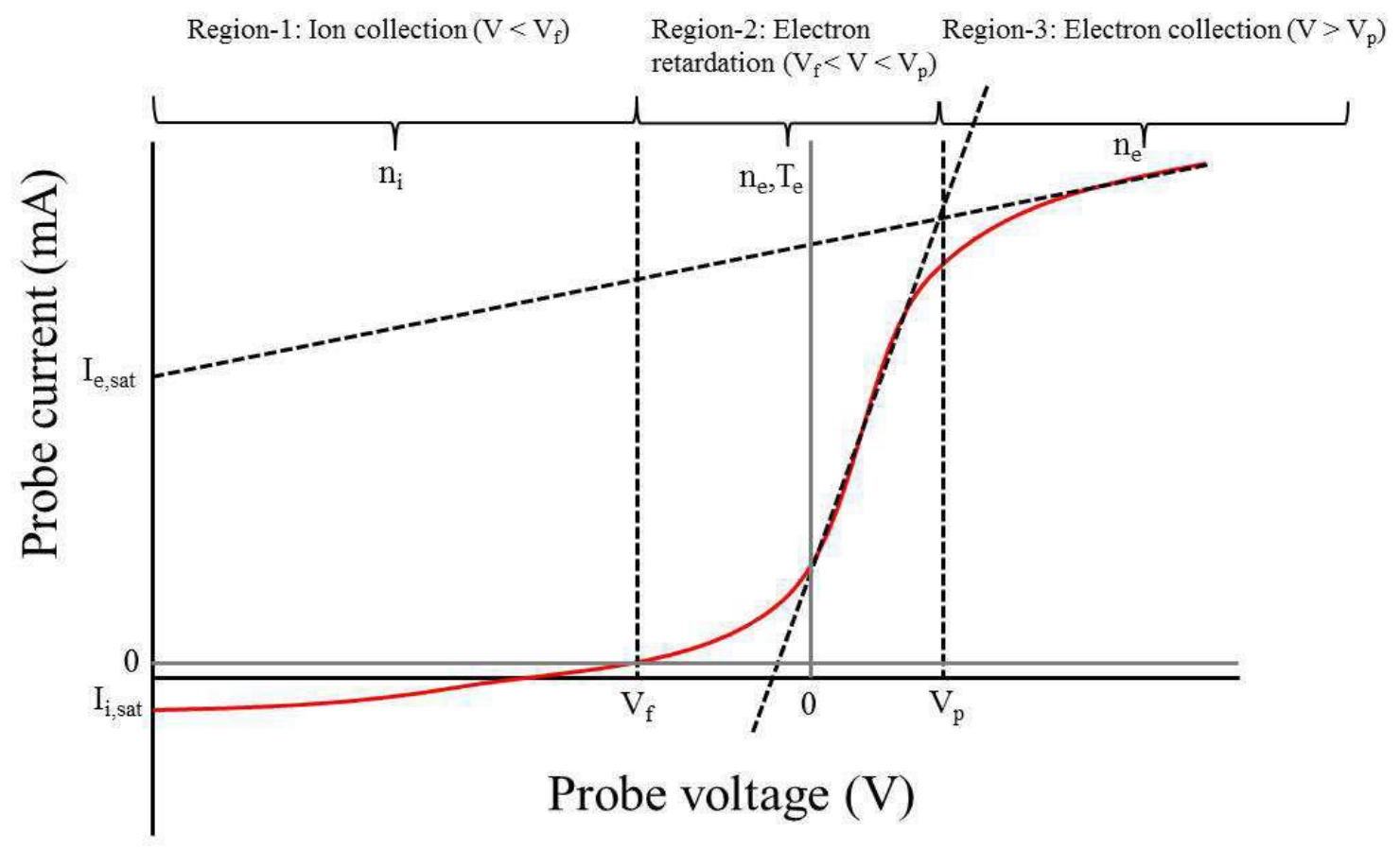

Figure 2: Current-voltage characteristics of Langmuir probe.

The typical trace of current-voltage characteristics with the use of Langmuir probe is shown in Fig. 2. It can be analyzed by considering three different regions as follows:

The region- 1 is corresponding to the values of the potential less than $V_{f}$ where floating potential $V_{f}$ is defined as the potential at which the electron and ion flux towards the probe comes in an equilibrium and hence the net current from the plasma is zero. Due to large mass of ions this current is generally small and negative. The drawn current is mainly contributed by positive ions and gives ion density in plasma.

The region-2 is corresponding to values of potential between $V_{f}$ and $V_{p}$, where $V_{p}$ is the plasma potential or space potential. At the beginning of this region from $V_{p}$, the electrons are initially attracted to the positive potential of the probe and hence form a sheath in order to shield the electric field produced. After the formation of sheath, the probe surface becomes negatively charged so it will start attracting the positive ions and repels the electrons to preserve the quasi neutrality of the plasma. Since the electrons are mainly retarded therefore this region is called electron retardation region. The retardation process continues until the ion and electron fluxes become equal at floating potential [1].

The region-3 describes the situation where the potential is positive and greater than $V_{p}$. Therefore, the electrons will be accelerated and positive ions will be repelled from the probe. Increasing the bias will yield the electron saturation current such that increase in voltage does not increase the current [10]. 


\section{Theory of Planar Langmuir Probe}

Although many plasma diagnostic methods have been developed since the original work of Irving Langmuir, the Langmuir probe is still one of the most powerful plasma diagnostic tools due to its simplicity, low cost, adaptability, ability to determine the fundamental characteristics of a plasma. A Langmuir probe may have any geometry, but cylindrical, spherical, and planar probes are usually employed because of their symmetry [11]. The basis for $T_{e}$ measurements is the conventional Langmuir probe theory of Mott-Smith and Langmuir, assuming a Maxwellian energy distribution $F(E)$ given by,

$$
F(E) \propto \sqrt{E} \exp \left(\frac{-E}{k T_{e}}\right)
$$

Where $k$ is the Boltzmann constant. Thus, in a Maxwellian plasma, when the probe to plasma potential, $V$, is driven negative, $I_{e}$ decreases exponentially.

The general formula of current in a charge plane conductor is given as,

$$
I_{\text {planar }} \square 1+\frac{V}{T}
$$

For $\mathrm{V}<0$,

$$
I_{e}=I_{e o} \exp \left(\frac{e V}{K T_{e}}\right)
$$

And

$$
I_{i}=I_{i o} \exp \left(1-\frac{e V}{K T_{i}}\right)
$$

While for $\mathrm{V}>0$,

$$
I_{e}=I_{e o} \exp \left(1+\frac{e V}{K T_{e}}\right)
$$

And

$$
I_{i}=-I_{i o} \exp \left(-\frac{e V}{K T_{i}}\right)
$$

Here the various currents are given as,

$$
I_{e o}=\operatorname{Ane}\left(\frac{K T_{e}}{2 \pi m_{e}}\right)^{1 / 2}
$$


Where $I_{\mathrm{eo}}$ is the random electron current, $e$ is the electron charge, $m_{e}$ is electron mass, and $A$ is the probe area. This equation for what is known as the electron retarding region in a Maxwellian plasma is the same for all probe geometries.

$$
\begin{aligned}
& I_{i o}=A n e\left(\frac{K T_{i}}{2 \pi m_{i}}\right)^{1 / 2} \\
& I_{e}=A n e\left(\frac{K T_{e}}{2 \pi m_{e}}\right)^{1 / 2} \exp \left(\frac{e V}{K T_{e}}\right)
\end{aligned}
$$

And for Planar Langmuir probe, $\mathrm{A}=2 \pi \mathrm{r}^{2}$

$$
I_{e}=2 \pi r^{2} n e\left(\frac{K T_{e}}{2 \pi m_{e}}\right)^{1 / 2} \exp \left(\frac{e V}{K T_{e}}\right)
$$

For a Maxwellian plasma, all three probe geometries are suitable for the measurement of $T_{e}$. However, the electron saturation regions differ greatly with collector geometry. The electron saturation region of the cylindrical probe is nearly independent of $T_{e}$, whereas this region is highly temperature dependent for the planar and spherical probes. The cylindrical and spherical probes have the advantage of producing large saturation currents for the same density, an advantage for measurements in regions of very low density.

\section{Results and Discussions}

The Langmuir probe technique for in situ measurement of plasma parameters has been around for eight decades, deriving the parameters with accuracy from the data acquired by a Langmuir probe immersed in space plasma is still a challenging task. In the present research work, theoretical study of Planar Langmuir Probe in Maxwellian plasma are studied. This work is typically based on the $I-V$ characteristic of Planar Langmuir Probe. The main propose of this work is to study the Current-Voltage characteristic of Planar Langmuir probe with different low ionospheric plasma temperatures. The methodology of our study includes the theoretical derivation of the relationships between various parameters and then plotting the parameters in suitable range from a mathematical software (here MATLAB is employed).

\section{The Variation of Planar Langmuir Probe I-V Curve with Temperatures}

The $I-V$ characteristic is a fundamental part of dagnosis of plasma parameters. So, it is depicted in the following figures. In the present study, the ionosphere parameters (electron temperature, ion temperature, electron density and ion density) are modelled. For this purpose, it is assumed that for density $N_{e}=10^{12} \mathrm{~m}^{-3}$, radius of planar probe $\left(\mathrm{r}_{\mathrm{p}}=2.5 \mathrm{~mm}\right)$. These figures illustrate the effect of $T_{e}$ on the width of the electron retradation regions. 
Here, Fig.3 depicts that the Planar Langmuir probe curves computed for a Maxwellian plasma illustrate the effect of Temperatures (i.e. $1 \mathrm{eV}, 2 \mathrm{eV}$ and $3 \mathrm{eV}$ ) respectively on the width of the retarding region. In order to obtain the $I-V$ curve the probe bias voltage is swept over a specified range $(-10 \mathrm{~V}$ to $+10 \mathrm{~V}$ in this simulation) that encompasses the plasma potential. The electron and ion currents collected by the probe will change depending on the applied bias voltage.

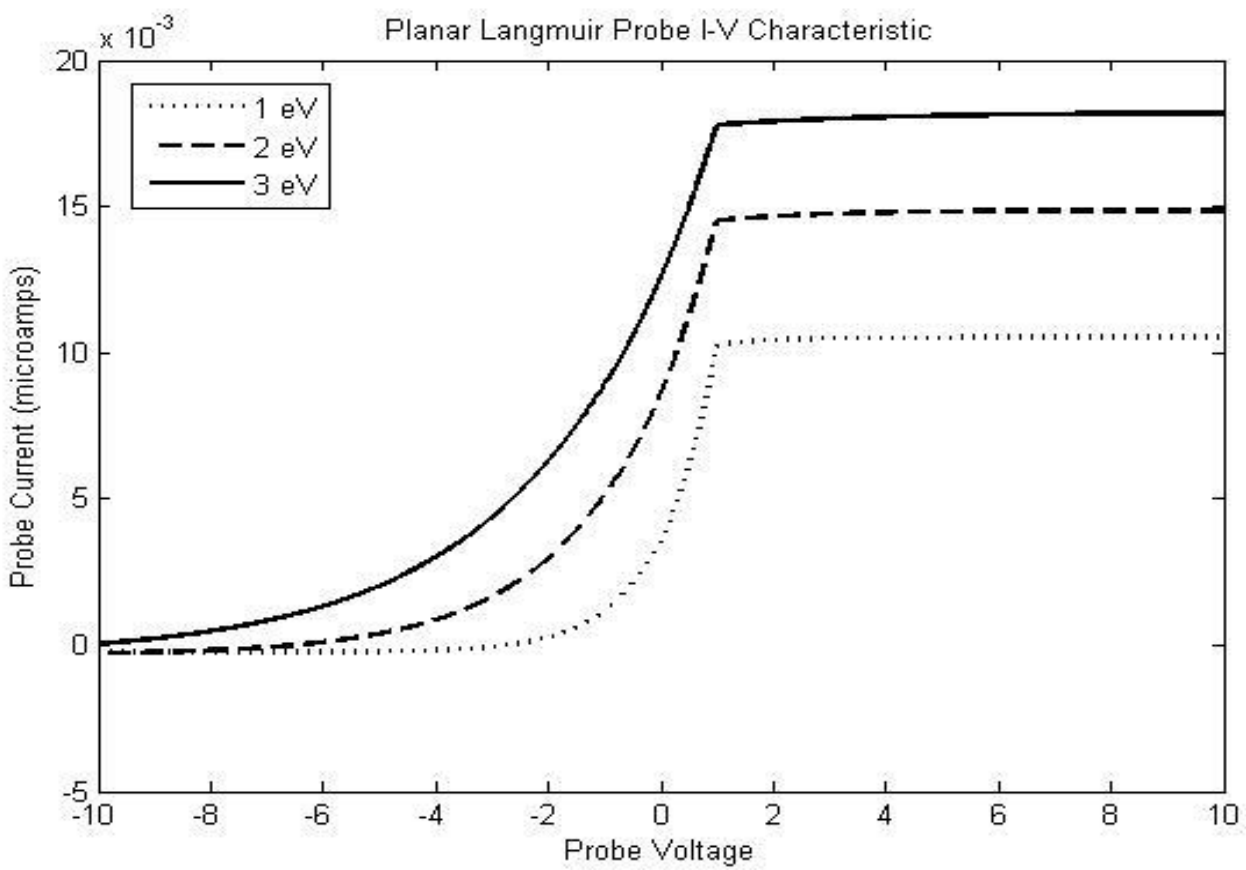

Figure 3: The Planar Langmuir Probe I-V curves computed for a Maxwellian plasma illustrate the effect of Te on the width of the retarding region.

The amplitude of the electron saturation current varies greatly with $T_{e}$. When the plasma temperature is $1 \mathrm{eV}$ the knee of the curve is sharp and is a good measure of $V_{\mathrm{s}}$. The Floating Potential $V_{f}$ is nearly -2 volt. This floating potential is typically negative because mobile electrons tend to strike the probe more frequently than positive ions. When the plasma temperature is $2 \mathrm{eV}$, the Floating Potential $V_{f}$ is nearly -6volt.

The knee occurs little bit sharper, when the probe has been saturated with electrons, causing additional electrons to be repelled. Plasma Potential $V_{s}$ is greater than 1 volt. At $3 \mathrm{eV}$, the Floating Potential $V_{f}$ is nearly -10 volts. For probe potential is greater than plasma potential $\left(V_{p}>V_{s}\right)$, electron saturation current is appearing constant due to an increase in sheath thickness. In the Transition Region, the ion current is negligible, and the electrons are partially repelled by the negative potential $V_{\mathrm{p}}-V_{\mathrm{s}}$. In a Maxwellian plasma, this part of the curve is exponential as shown in above figure.

\section{Derivative of I-V characteristics of Planar Langmuir Probe}

The Figure below shows the first derivative of $I-V$ characteristics of planar Langmuir probe in Maxwellian plasma. All three graphs shows the relation between derivative of current versus bias voltage and probe current verses applied probe voltage. 


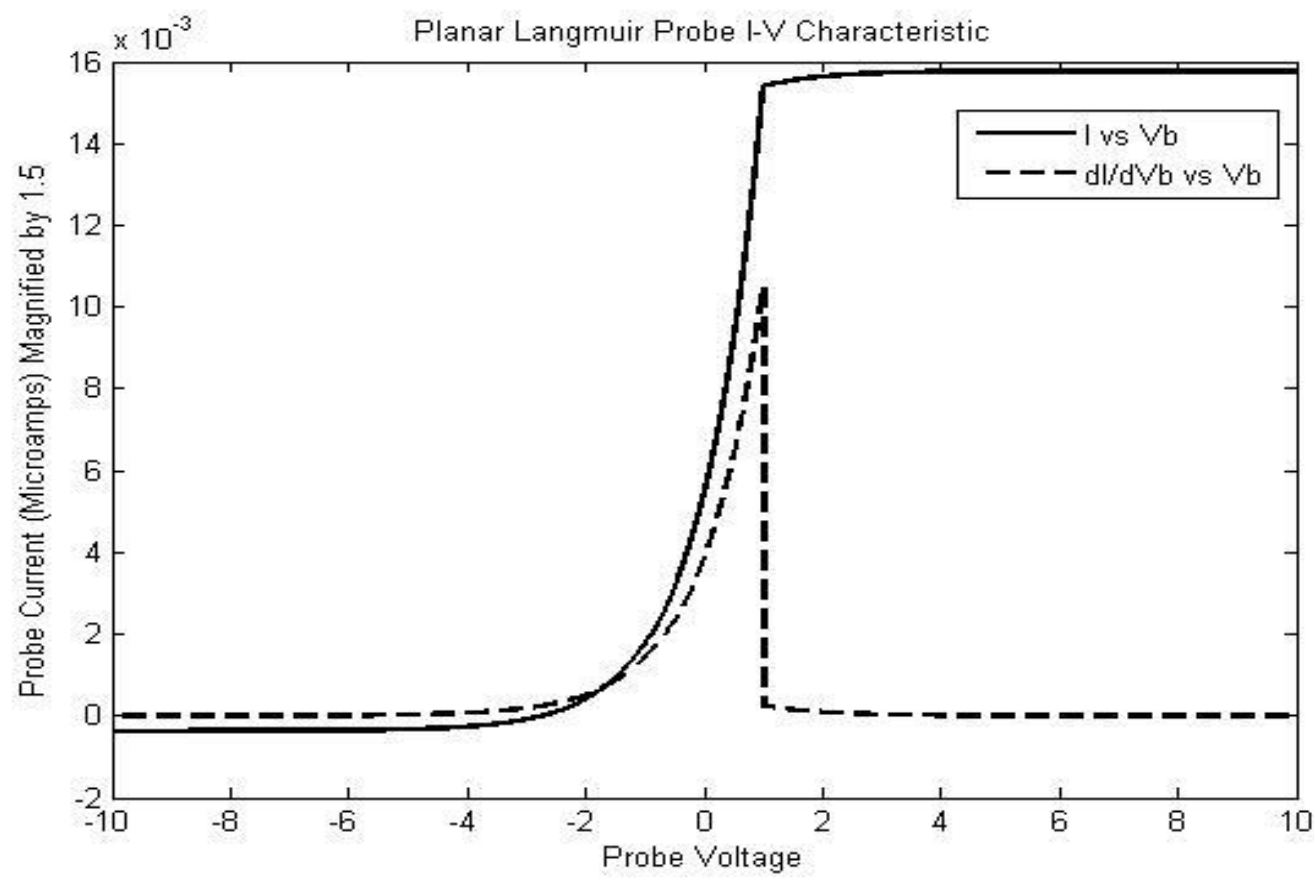

Figure 4: The Planar Langmuir Probe I-V curve computed for a Maxwellian plasma with first derivative at $\mathrm{Te}=1 \mathrm{eV}$.

In Fig. 4, the first derivative of the probe electron current $I_{e}$ versus the probe voltage $V_{p}$ provides a peak value of $1 \mathrm{~V}$, which corresponds to the space potential (plasma potential) when the electron temperature is $1 \mathrm{eV}(11600 \mathrm{~K})$. The negative ions and positive ions saturation currents are comparable, with such characteristic the plasma potential is easily determined as the voltage at which the first derivative is maximum.

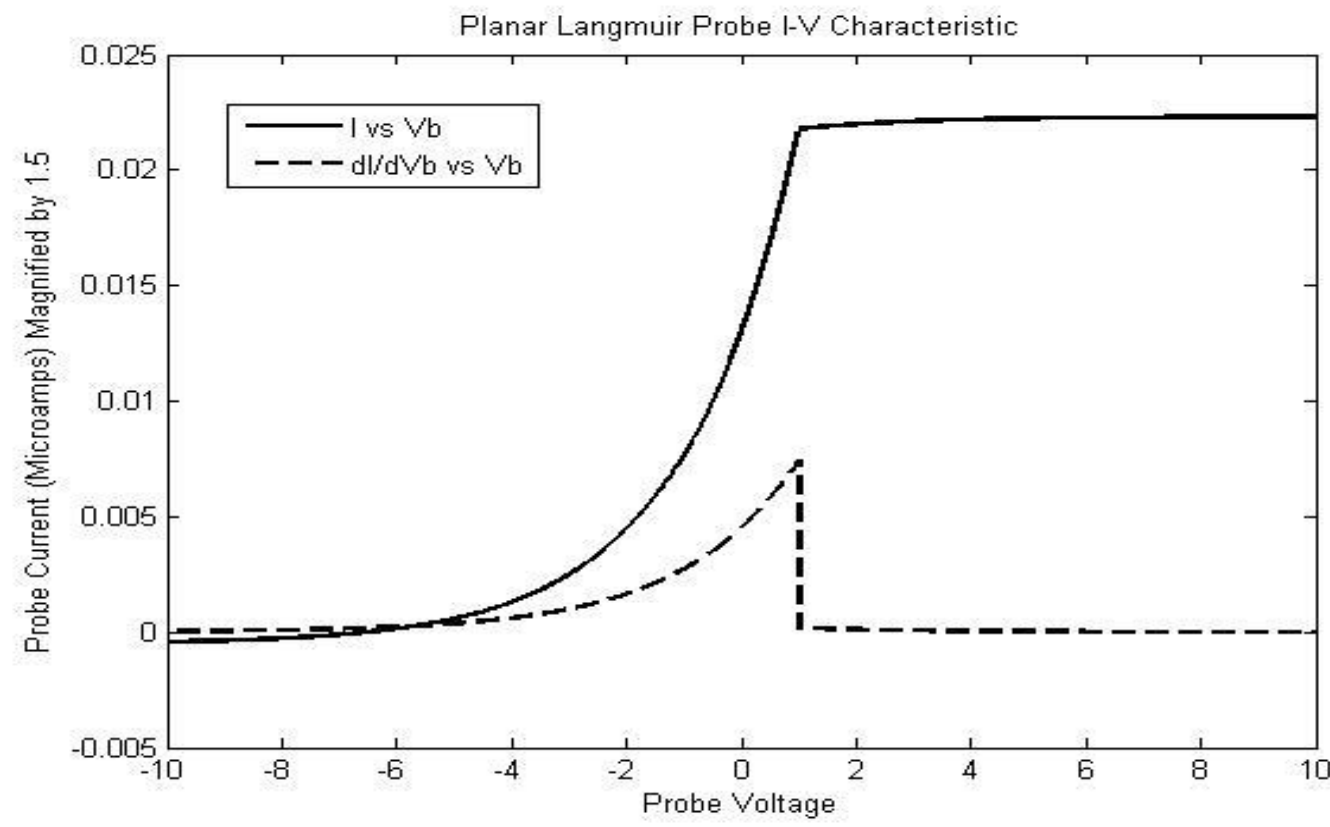

Figure 5: The Planar Langmuir Probe I-V curve computed for a Maxwellian plasma with first derivative at $\mathrm{Te}=2 \mathrm{eV}$. 
Here in Fig.5 shows that, when the electron temperature is $2 \mathrm{eV}(23200 \mathrm{~K})$, the point of inflection of $I-V$ characteristics i.e. $d I / d V$ is maximum corresponds to $1 \mathrm{~V}$ plasma potential. So here the plasma potential is $1 \mathrm{~V}$, which is expected result and also that the characteristics is roughly symmetric about $\mathrm{I} \approx 8 \mathrm{~mA}$.

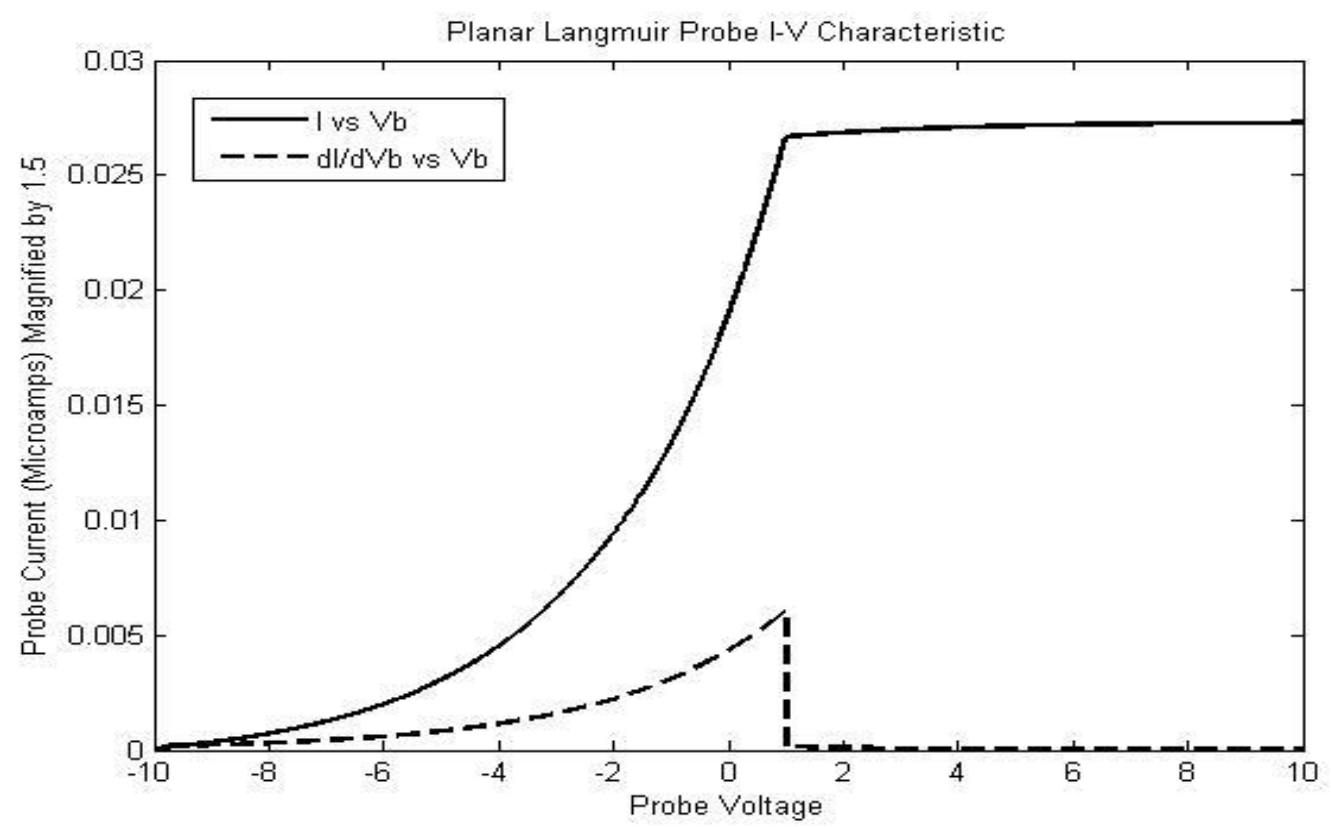

Figure 6: The Planar Langmuir Probe I-V curve computed for a Maxwellian plasma with first derivative at $\mathrm{Te}=3 \mathrm{eV}$.

Finally, the graph shown in Fig.4, Fig.5, and Fig.6 are outcomes of the plot $I-V$ curves and its first derivative with probe voltage and first derivative shows the plasma potential. In all three graphs the plot of derivative is represented by the pulse seen distinctly. The $I-V$ curves were plotted at different value of plasma potential with remaining ion or electron temperature constant.

\section{Conclusion}

This research has attempted to improve the application of planar Langmuir probes to unmagnetized collisionless plasma. The physical conditions under which the presented model is valid and can be applied have been discussed. The $I-V$ characteristic of the Planar Langmuir Probe in the Maxwellian Space plasma at different temperature range are plotted in graph by using the mathematical software by putting the suitable ranges for various parameters. All elementary processes were extensively discussed and most of information has been presented. With the help of the (volt-ampere curves) of Planar Probes, the different parameters of plasma can be determined such as plasma potential, floating potential, probe currents in different probe voltage and so on. And the effect of temperature $T_{e}$ on the width of the electron retardation regions are clearly studied in this research. Thus, measured plasma parameters, and the relationship between the density of the plasma on probe current, effect of temperature on $I-V$ curve, are of fundamental importance for research on laboratory plasma, ionosphere terrestrial 
plasma and industrial application. The first derivative of current voltage characteristic is suitable for to determine the plasma potential.

\section{References}

[1] Langmuir, and H.M. Mott-Smith, "The theory of collectors in Gaseous Discharges," Physical Review, vol. 20, p. 727, 1926.

[2] F. F. Chen, "Electric Probes," Plasma Diagnostic Techniques, pp. 113-200, 1965.

[3] T. N. Academies, Plasma Science: From Fundamental Research to Technological Applications, National Academy Press, 1995.

[4] ImpedansLtd, Plasma Management to Understand and Control the Future, http:/www.impedans.com/langmuir probes, 2014.

[5] J. G. Laframboise, "Theory of spherical and cylindrical Langmuir probes in a collisionless, Maxwellian plasma at rest," UTIAS, 1966.

[6] J. G. Laframboise, Theory of Spherical and Cylindrical Langmuir Probes in a Collisionless, Maxwellian Plasma at Rest, Toronto: UTIAS Report No.100 University of Toronto, 1966.

[7] L. Conde, An introduction of Langmuir probe diagnostics of plasmas, Madrid: Universidad Politecnica de Madrid, 2011.

[8] D. Bohm, E. H. S. Burhop, and H. S. W. Massey, The Characteristics of Electrical Discharges in Magnetic Fields, New York: McGraw Hill, New York, 1949.

[9] Piel, M. Hirt, and C. T. Steigies, "Plasma diagnostics with Langmuir probes in the equatorial ionosphere: I. The influence of surfacecontamination," J. Phys. D: Appl. Phys., vol. 34, no. 17, pp. 2643-2649, 2001.

[10] W.E. Amatucci, P. W. Schuck, D. N. Walker, P. M. Kintner, S. Powell, B. Holback, and D. Leonhardt, "Contamination free sounding rocket Langmuir probe," Rev. Sci. Instrum., vol. 72, no. 4, p. 2052, 2001.

[11] V.N. Rai, M. Shukla, H.C. Pant and D.D. Bhawalkar, "High Speed Plasma Diagnostics for laser plasma interaction and fusion studies," Sudhana, vol. 24, p. 513, 1999.

[12] P. M. Bellan, Fundamentals of Plasma Physics, Cambridge: Cambridge University Press, 2008.

*Corresponding author.

E-mail address: oshobhattarai2@gmail.com 